\title{
CDISC SEND Chemical Challenge Agent Name Response Terminology
}

National Cancer Institute

\section{Source}

National Cancer Institute. CDISC SEND Chemical Challenge Agent Name Response

Terminology. NCI Thesaurus. Code C160930.

Terminology associated with the chemical challenge agent name response codelist of the Clinical Data Interchange Standards Consortium (CDISC) Standard for the Exchange of Non-clinical Data (SEND). 